\title{
Fully Printed Halide Perovskite Light-Emitting Diodes with Silver Nanowire Electrodes
}

Sri Ganesh R. Bade ${ }^{1}$, Junqiang Li $^{1}$, Xin Shan ${ }^{1}$, Yichuan Ling ${ }^{2}$, Yu Tian ${ }^{5}$, Tristan Dilbeck ${ }^{4}$, Tiglet Besara $^{6}$, Thomas Geske, ${ }^{1,5}$, Hanwei Gao ${ }^{2,5}$, Biwu Ma ${ }^{3,4,5}$, Kenneth Hanson ${ }^{4,5}$, Theo Siegrist ${ }^{3,5,6}$, Chengying $\mathrm{Xu}^{7}$ and Zhibin $\mathrm{Yu}^{1,5 *}$

1. Department of Industrial and Manufacturing Engineering, High Performance Materials Institute, Florida State University, Tallahassee FL 32310, USA

2. Department of Physics, Florida State University, Tallahassee FL 32306, USA

3. Department of Chemical and Biomedical Engineering, Florida State University, Tallahassee FL 32310, USA

4. Department of Chemistry and Biochemistry, Florida State University, Tallahassee FL 32306, USA

5. Materials Science and Engineering, Florida State University, Tallahassee FL 32306, USA

6. National High Magnetic Field Laboratory, Tallahassee FL 32310, USA

7. Department of Mechanical Engineering, Florida State University, Tallahassee FL 32310, USA

*Email: zyu@fsu.edu 
a)

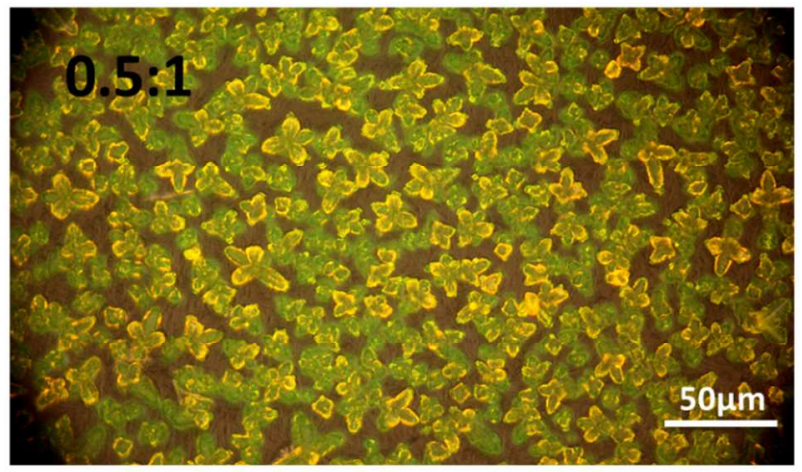

b)

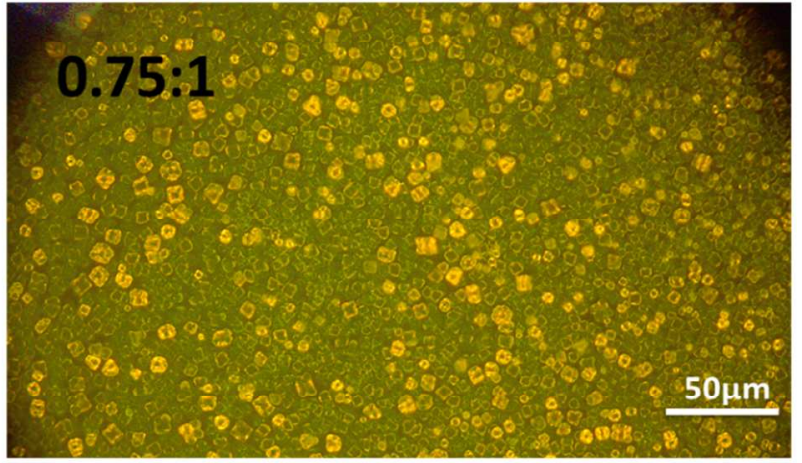

c)

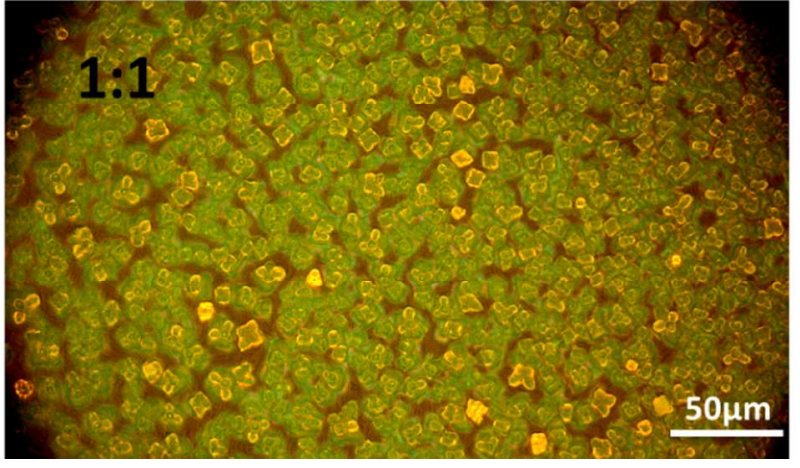

Figure S1. Optical microscopic images of the printed PEO/Br-Pero films with PEO to Pero weight ratio of a) $0.5: 1$, b) $0.75: 1$ and c) $1: 1$. 


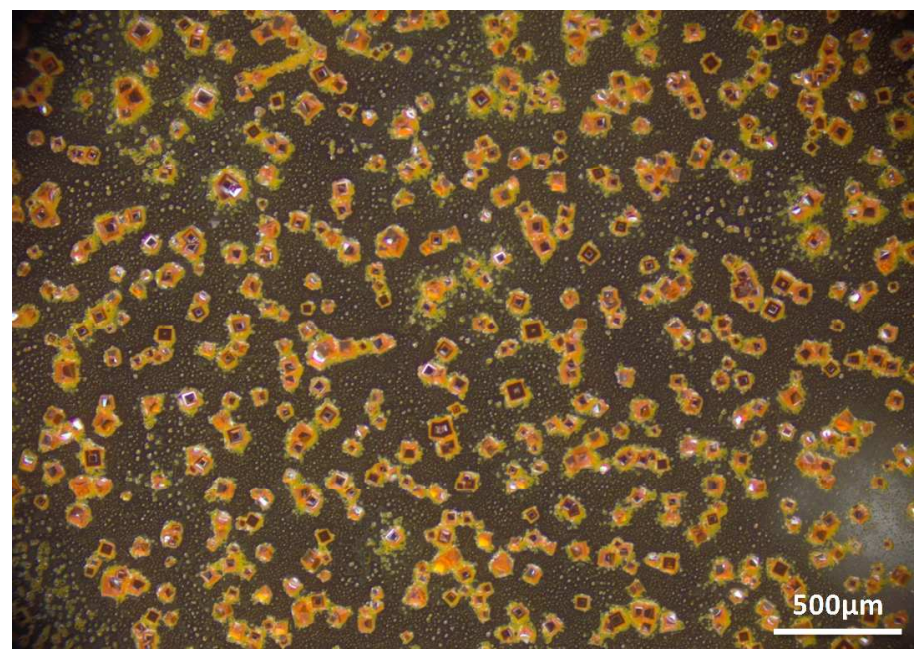

Figure S2. An optical microscopic image of a Br-Pero film printed in air without adding PEO.

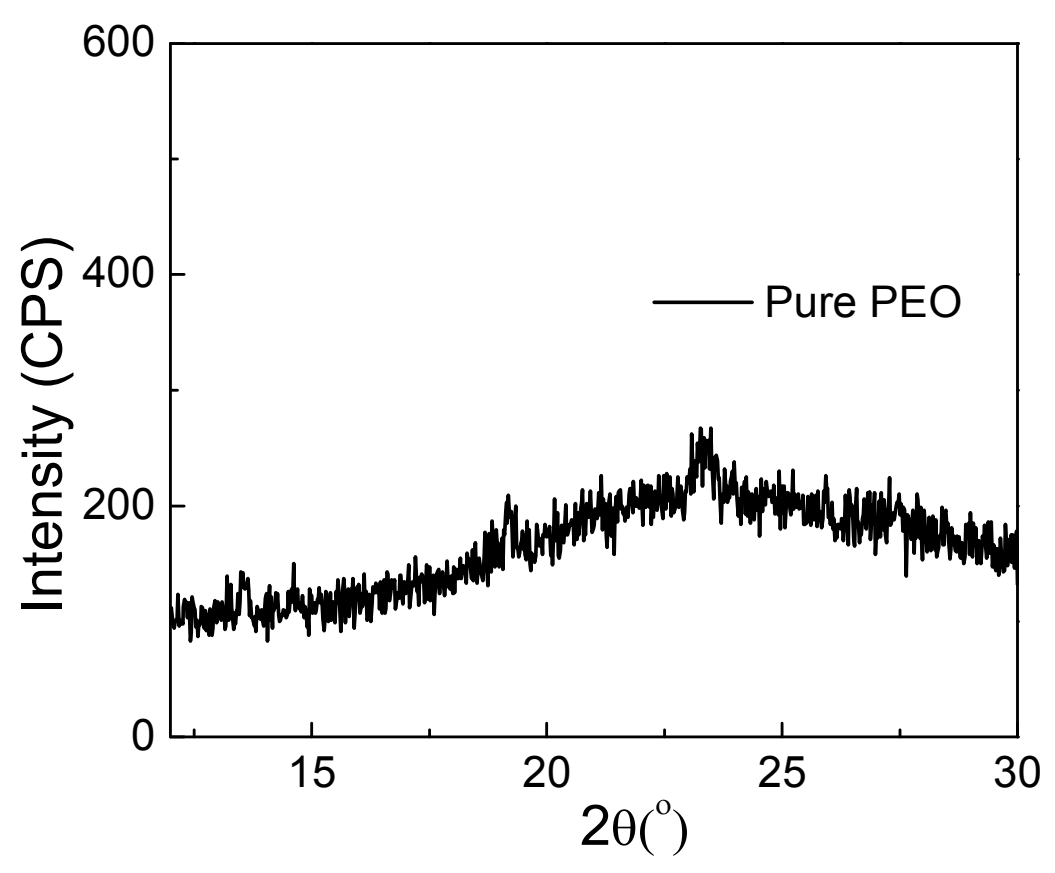

Figure S3. X-ray diffraction pattern of a pure PEO film 
Ohr

a)

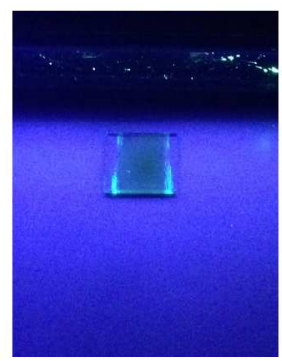

$8 \mathrm{hr}$

b)

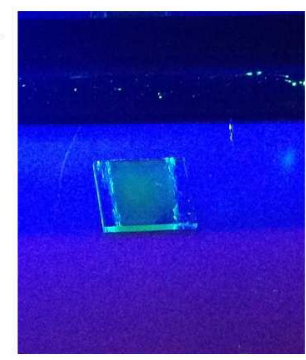

$32 \mathrm{hr}$

c)

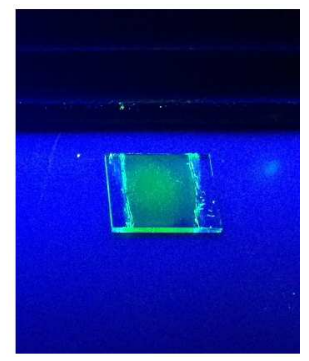

Figure S4. Photos of one PEO/Br-Pero composite film (0.75:1) under 365-nm UV lamp irradiation. a) Immediately after printing inside a nitrogen filled glovebox, b) after 8 hours, and c) after 32 hours inside the glovebox.

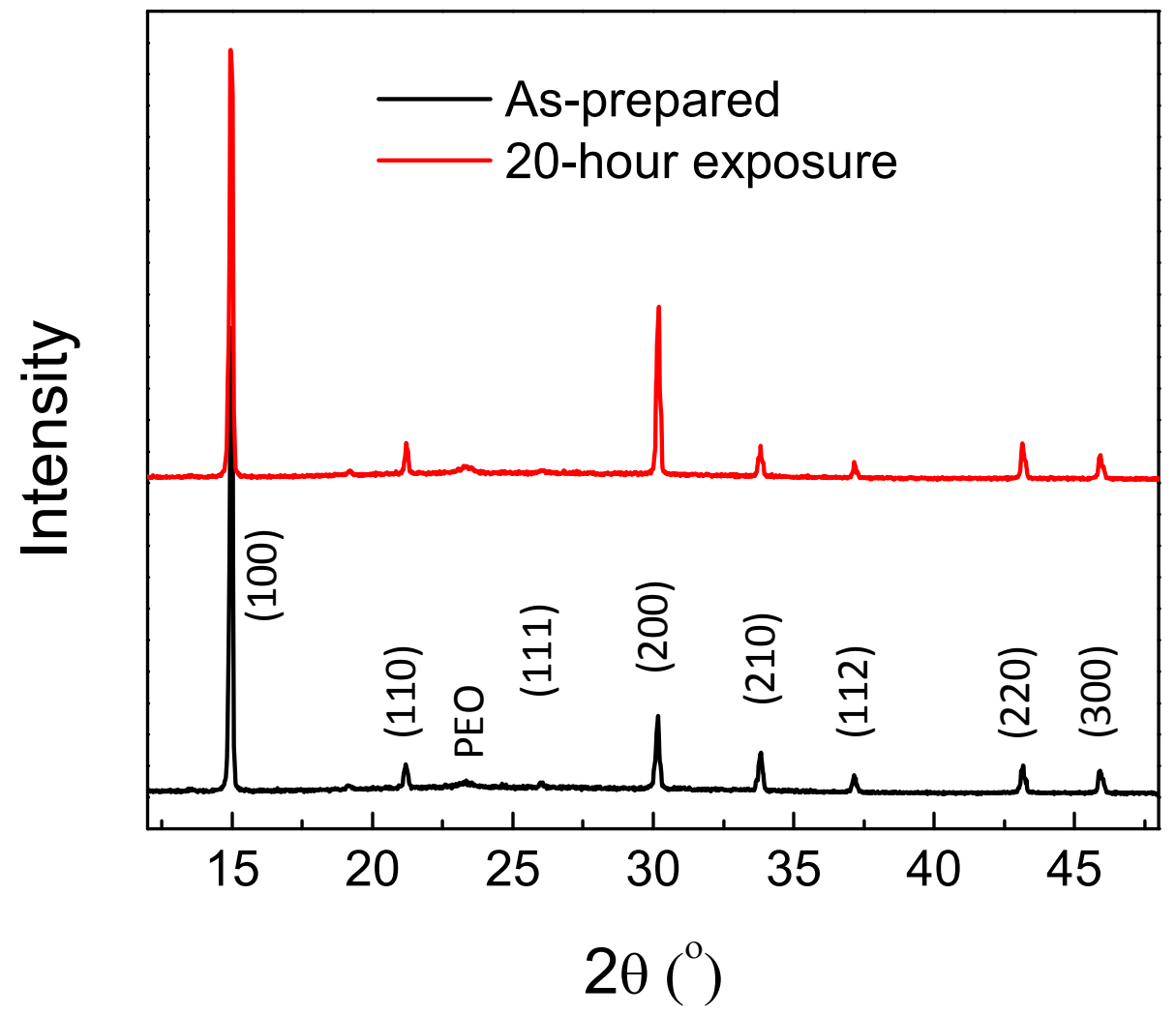

Figure S5. X-ray diffraction patterns of one PEO/Br-Pero composite film (0.75:1). a) Immediately after printing in air and, b) after 20 hours exposure in air. 
a)

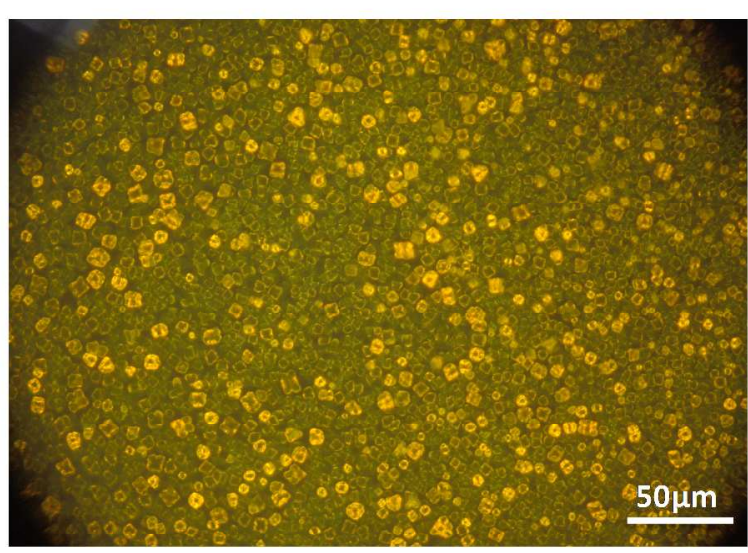

As-prepared

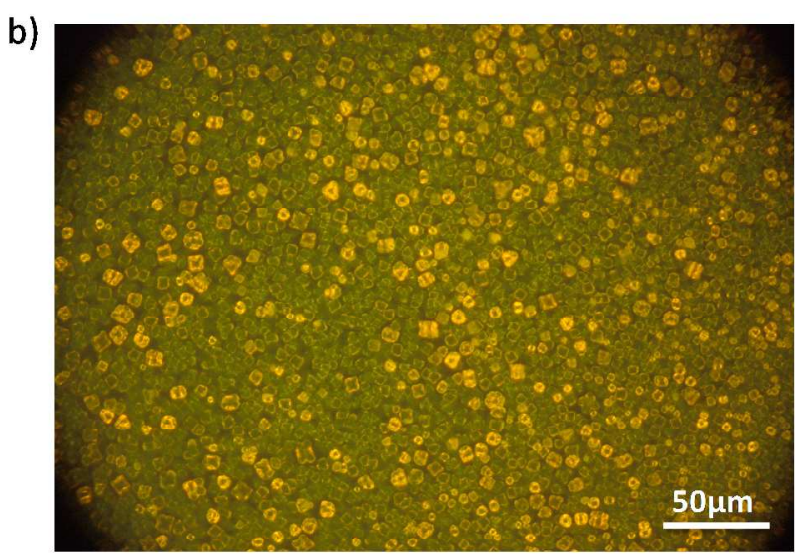

After $\mathbf{2 0}$ hours air exposure

Figure S6. Optical microscopic images of one PEO/Br-Pero composite film (0.75:1). a) Immediately after printing in air, and b) after 20 hours exposure in air.

Table S1. Average lifetimes for perovskites with 0 and 25 hour exposure to air.

\begin{tabular}{cccc}
\hline \multirow{2}{*}{$\begin{array}{c}\text { Exposure } \\
\text { time } \\
(\mathrm{hrs})\end{array}$} & \multicolumn{3}{c}{ lifetime (ns) } \\
\cline { 2 - 4 }$\tau_{1}\left(\mathrm{~A}_{1}\right)$ & $\tau_{2}\left(\mathrm{~A}_{2}\right)$ & $<\tau>$ \\
\hline $\mathbf{0}$ & $162(77)$ & $477(23)$ & 309 \\
$\mathbf{2 5}$ & $478(71)$ & $1226(29)$ & 861 \\
\hline
\end{tabular}

Time-resolved emission data were fit by using the bi-exponential function in Equation S1. A weighted average of lifetime $\tau$ is calculated by using Equation S2.

$$
\begin{gathered}
\mathrm{y}=\mathrm{A}_{1} \mathrm{e}_{1}^{-\mathrm{k}{ }_{1}}+\mathrm{A}_{2} \mathrm{e}_{2}^{-\mathrm{k} x}+\mathrm{y}_{0} \\
\tau_{\mathrm{i}}=1 / \mathrm{k}_{\mathrm{i}} \quad ;<\tau>=\Sigma \mathrm{A}_{\mathrm{i}} \tau_{\mathrm{i}}^{2} / \Sigma \mathrm{A}_{\mathrm{i}} \tau
\end{gathered}
$$



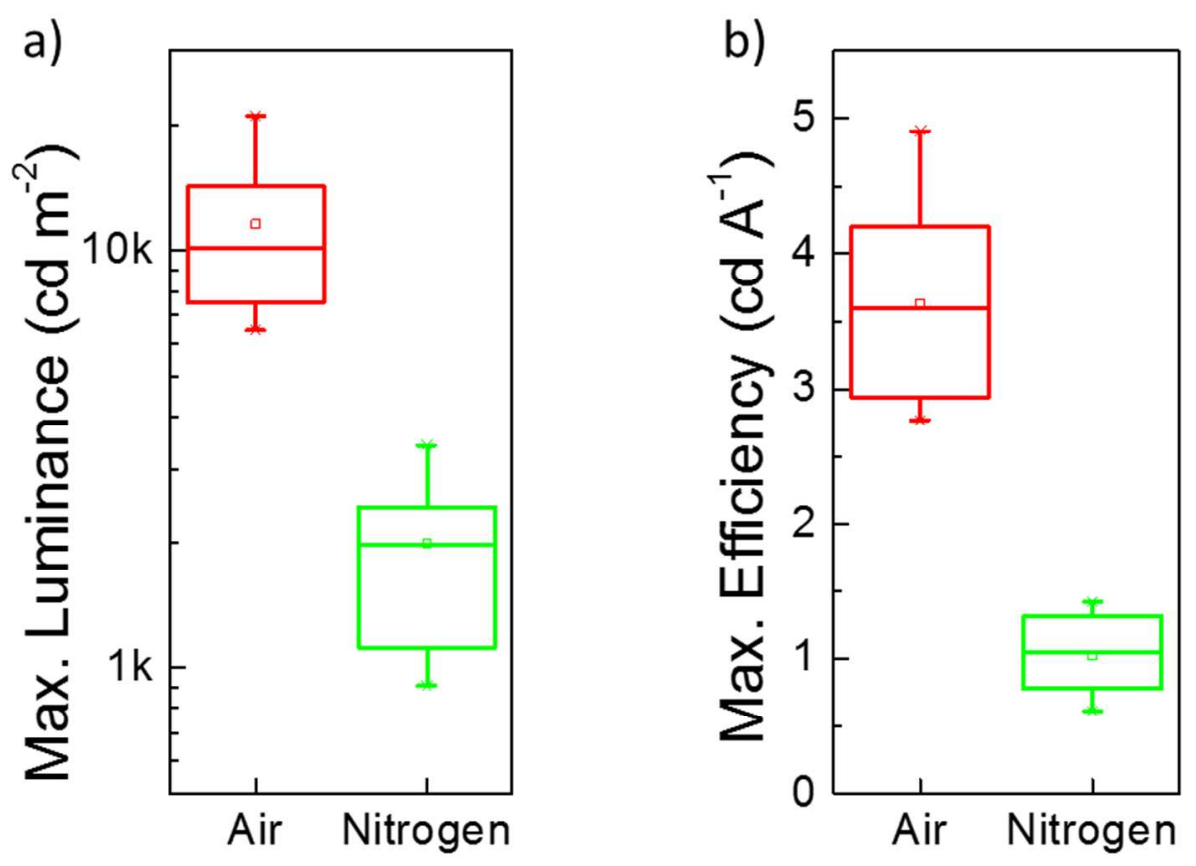

Figure S7. a) Statistical summaries of $L_{\max }$ and, b) $E_{\max }$ for seven devices from the same batch of fabrication in air and in the nitrogen filled glovebox. 